\title{
A Note on Bi-Orthogonal Polynomials and Functions
}

\author{
Clemente Cesarano
}

Section of Mathematics, Uninettuno University, 00186 Roma, Italy; c.cesarano@uninettunouniversity.net

Received: 2 May 2020; Accepted: 25 June 2020; Published: 30 June 2020

\begin{abstract}
The theory of orthogonal polynomials is well established and detailed, covering a wide field of interesting results, as, in particular, for solving certain differential equations. On the other side the concepts and the related formalism of the theory of bi-orthogonal polynomials is less developed and much more limited. By starting from the orthogonality properties satisfied from the ordinary and generalized Hermite polynomials, it is possible to derive a further family (known in literature) of these kind of polynomials, which are bi-orthogonal with their adjoint. This aspect allows for us to introduce functions recognized as bi-orthogonal and investigate generalizations of families of orthogonal polynomials.
\end{abstract}

Keywords: Hermite polynomials; generating functions; bi-orthogonal polynomials; bi-orthogonal functions

\section{Introduction}

The topic of bi-ortogonality will be treated while using the formalism and the operational properties satisfied by different classes of polynomials recognizable as generalized Hermite polynomials $[1,2]$. The consolidated approach to the study of the characteristics of orthogonality and, although less developed, the one related to the concept of bi-ortogonality will be reread on the different formalisms that can be obtained from the various relations deducible from the structure of the different polynomials and the related functions that are attributable to the family of Hermite polynomials [3-5]. The fulcrum of the discussion is based on the two-dimensional extension of Hermite polynomials

$$
H_{n}(x) \rightarrow H_{(m, n)}(x, y),
$$

where we consider the variables as real and the indexes positive integers.

\section{Orthogonality and Bi-Orthogonality}

By starting from the space $L_{w}^{2}(a, b)$ and by considering a real function $w(x)$ on the interval $(a, b)$, non-negative, measurable, and non-zero, we can define the inner product (if for every $f(x) \in$ $L_{w}^{2}(a, b), f(x)^{2} w(x)$ is Lebesgue integrable):

$$
<f, g>\stackrel{\text { def }}{=}(f, g)_{w}:=\int_{a}^{b} f(x) g(x) w(x) d x,
$$

which define an Hilbert space. The functions $f$ and $g$ will be said orthogonal, if:

$$
<f, g>=0
$$

and the function $w(x)$ is usually referred as the weight function. 
Remark 1. For our purpose, it is not necessary to introduce the concept of orthogonality through the Lebesgue-Stieltjes integral, by introducing a general measure $m(x)$ on $\mathbb{R}$, by assuming that:

$$
x^{k} \in L_{w}^{2}(a, b), \forall k \in \mathbb{N}
$$

i.e., the moment sequence $\mu_{k}, k=0,1, \ldots$ finite, $\mu_{k}:=\int_{a}^{b} x^{k} w(x) d x$, with $w(x)>0$.

Under these hypothesis, we can construct the Hankel determinant, which allows for us to define the family of orthogonal polynomials by the following:

$$
\left\{\begin{array}{c}
P_{0}(x)=\mu_{0}^{-\frac{1}{2}} \\
P_{n}(x)=\frac{1}{\sqrt{H_{n} H_{n+1}}}\left|\begin{array}{ccc}
\mu_{0} & \ldots & \mu_{n} \\
\ldots & \ldots & \ldots \\
1 & \ldots & x^{n}
\end{array}\right|
\end{array}\right.
$$

where

$$
H_{n+1}=\left|\begin{array}{ccc}
\mu_{0} & \ldots & \mu_{n} \\
\ldots & \ldots & \ldots \\
\mu_{n} & \ldots & \mu_{2 n}
\end{array}\right|
$$

In a more direct way, a sequence of polynomials $\left\{P_{n}(x)\right\}_{n=0}^{+\infty}$ is said orthogonal if, for any $n, m \in \mathbb{N}$, we have:

(i) $P_{n}(x)$ is a polynomial of exactly degree $n$

(ii) $\left\langle P_{n}, P_{m}\right\rangle=K_{n} \delta_{n, m}$

$K_{n}>0, \delta_{n, m}$ denotes the Kronecker delta. The sequence is said to be orthonormal if:

(ii)' $<P_{n}, P_{m}>=\delta_{n, m}$

To introduce the bi-orthogonality, we need to define a totally positive kernel [6]; that is a two-variable continuous real function $K(x, y)$, such that, for all:

$$
x_{1}<x_{2}<\ldots<x_{m}, y_{1}<y_{2}<\ldots<y_{m}
$$

it holds that

$$
\operatorname{det}\left[K\left(x_{i}, y_{i}\right)\right]_{1 \leq i, j \leq m}>0
$$

By assuming the above condition and, furthermore, that the integrals involved are defined and finite, we can define an inner (bi-)product.

Let $p, q \in \mathcal{P}$, we said, non-symmetric inner (bi-)product, the relation:

$$
<p(x), q(x)>:=\iint_{\mathcal{D}} p(x) q(y) K(x, y) d x d y
$$

where $\mathcal{P}$ is the space of real polynomials, $\mathcal{D} \neq \varnothing, \mathcal{D} \subseteq \mathbb{R}^{2}$

To better understand the nature of bi-orthogonality, we set the following.

Definition 1. Two sequences of polynomials $\left\{P_{n}(x)\right\}_{n=0}^{+\infty}$ and $\left\{Q_{n}(x)\right\}_{n=0}^{+\infty}$ are said to be bi-orthogonal polynomial sequences, if for any $n, m \in \mathbb{N}$, we have:

(i) $P_{n}(x)$ and $Q_{m}(x)$ are polynomials of exactly degree $n$ and $m$, respectively

(ii) $<P_{n}, Q_{m}>=K_{n} \delta_{n, m}, K_{n}>0$

If $K_{n}=1$ the sequences are said bi-orthonormal. 
Remark 2. In general, since the above definition, we have:

$$
<P_{n}, Q_{m}>\neq<Q_{m}, P_{n}>
$$

This standard definition of bi-orthogonality is strictly based on the concept of orthogonality [6-8]. It is useful to note that, with Cauchy kernel:

$$
1 / x+y
$$

we obtain biorthogonal polynomials, called Cauchy biorthogonal polynomials, which appear in the study of special solutions to the dispersive non-linear partial differential equation, called the Degasperis-Procesi (DP) equation, which represents an approximate model for wave propagation [9].

In the next sections, we will describe the bi-orthogonality relations for a special class of Hermite polynomials, which can be seen as a particular case of the previous definition, but from the other side, as a first step to generalize the concept itself in a different context.

\section{Hermite Polynomials and Related Functions}

We start to introduce the ordinary one-variable Hermite polynomials and their orthogonal properties, by setting their explicit form $([10,11])$ :

$$
H_{n}(x)=n ! \sum_{r=0}^{[n / 2]} \frac{(-1)^{r} x^{(n-2 r)}}{r !(n-2 r) ! 2^{r}}
$$

and the related generating function, which reads:

$$
\exp \left(x t-\frac{t^{2}}{2}\right)=\sum_{n=0}^{+\infty} \frac{t^{n}}{n !} H_{n}(x)
$$

The ordinary Hermite polynomials are orthogonal on the interval $(-\infty,+\infty)$, with respect to the weight function:

$$
e^{-\frac{x^{2}}{2}}
$$

i.e.,

$$
\int_{-\infty}^{+\infty} H_{n}(x) H_{m}(x) e^{-\frac{x^{2}}{2}} d x=n ! \sqrt{2 \pi} \delta_{n, m}
$$

The above relation suggests dor us to introduce a family of functions, based on the ordinary Hermite themselves in such a way as similar properties are derived, we set $[12,13]$ :

$$
h e_{n}(x)=\left(\frac{1}{\sqrt{2 \pi} n !}\right)^{\frac{1}{2}} H_{n}(x) e^{-\frac{x^{2}}{4}}
$$

which are orthonormal on the interval $(-\infty,+\infty)$ :

$$
\int_{-\infty}^{+\infty} h e_{n}(x) h e_{m}(x) d x=\delta_{n, m}
$$

The one-variable orthogonal Hermite functions $h e_{m}(x)$ satisfy the following recurrence relations:

$$
\begin{gathered}
2 \frac{d}{d x} h e_{m}(x)=\sqrt{m} h e_{m-1}(x)-\sqrt{m+1} h e_{m+1}(x), \\
x h e_{m}(x)=\sqrt{m} h e_{m-1}(x)+\sqrt{m+1} h e_{m+1}(x) .
\end{gathered}
$$


In fact, by deriving with respect to $x$ both sides of the relation defining $h e_{m}(x)$, we have:

$$
\frac{d}{d x} h e_{m}(x)=\left(\frac{1}{\sqrt{2 \pi} m !}\right)^{\frac{1}{2}} \frac{d}{d x}\left(H e_{m}(x) e^{-\frac{x^{2}}{4}}\right)
$$

and by using the recurrence relation satisfied by the Hermite polynomials, we can write:

$$
\frac{d}{d x} h e_{m}(x)=\left(\frac{1}{\sqrt{2 \pi} m !}\right)^{\frac{1}{2}}\left[m H e_{m-1}(x) e^{-\frac{x^{2}}{4}}-\frac{x}{2} H e_{m}(x) e^{-\frac{x^{2}}{4}}\right] .
$$

Futhermore, it is easy to prove that the ordinary Hermite polynomial $\mathrm{He}_{m}(x)$ satisfies the following relation:

$$
H e_{m}(x)=\frac{1}{x}\left[m H e_{m-1}(x)+H e_{m+1}(x)\right]
$$

which helps us to write the previous relation in the following form:

$$
\frac{d}{d x} h e_{m}(x)=\left(\frac{1}{\sqrt{2 \pi} m !}\right)^{\frac{1}{2}}\left[m H e_{m-1}(x) e^{-\frac{x^{2}}{4}}-\left(\frac{1}{2} e^{-\frac{x^{2}}{4}}\left(m H e_{m-1}(x)+H e_{m+1}(x)\right)\right)\right] .
$$

By substituting the expression of the Hermite polynomials $\mathrm{He}_{m-1}(x)$ and $H e_{m+1}(x)$ in terms of the orthogonal Hermite functions $h e_{m}(x)$, we obtain:

$$
\begin{aligned}
& \frac{d}{d x} h e_{m}(x)= \\
& \quad\left(\frac{1}{\sqrt{2 \pi} m !}\right)^{\frac{1}{2}}\left[m e^{-\frac{x^{2}}{4}}(\sqrt{2 \pi}(m-1) !)^{\frac{1}{2}} e^{\frac{x^{2}}{4}} h e_{m-1}(x)+\right. \\
& \left.\quad-\frac{1}{2} e^{-\frac{x^{2}}{4}} n(\sqrt{2 \pi}(m-1) !)^{\frac{1}{2}} e^{\frac{x^{2}}{4}} h e_{m-1}(x)-\frac{1}{2} e^{-\frac{x^{2}}{4}}(\sqrt{2 \pi}(m+1) !)^{\frac{1}{2}} e^{\frac{x^{2}}{4}} h e_{m+1}(x)\right]
\end{aligned}
$$

and then:

$$
\frac{d}{d x} h e_{m}(x)=\frac{m[(m-1) !]^{\frac{1}{2}}}{(m !)^{\frac{1}{2}}} h e_{m-1}(x)-\frac{1}{2} \frac{m[(m-1) !]^{\frac{1}{2}}}{(m !)^{\frac{1}{2}}} h e_{m-1}(x)-\frac{1}{2} \frac{m[(m+1) !]^{\frac{1}{2}}}{(m !)^{\frac{1}{2}}} h e_{m+1}(x)
$$

which proves the first of recurrence relation. Regarding the second relation, we start to note that the Hermite polynomials $\mathrm{He}_{m}(x)$ satisfy the following recurrence relation:

$$
x H e_{n}(x)=H e_{n+1}(x)+n H e_{n-1}(x)
$$

and, by substituting the expressions of the ordinary Hermite polynomials in terms of the related Hermite functions, we immediately obtain:

$$
\begin{aligned}
& x\left[(\sqrt{2 \pi} m !)^{\frac{1}{2}} e^{\frac{x^{2}}{4}} h e_{m}(x)\right]= \\
& \quad=(\sqrt{2 \pi}(m+1) !)^{\frac{1}{2}} e^{\frac{x^{2}}{4}} h e_{m+1}(x)+m(\sqrt{2 \pi}(m-1) !)^{\frac{1}{2}} e^{\frac{x^{2}}{4}} h e_{m-1}(x)
\end{aligned}
$$

which prove the second recurrence relations. 
In order to explore the differential characteristics involving the orthogonal Hermite functions of the type $h e_{m}(x)$, we can use the above recurrence relation, which, properly manipulated, gives us the following:

$$
\begin{aligned}
& \left(\frac{d}{d x}+\frac{x}{2}\right) h e_{m}(x)=\sqrt{m} h e_{m-1}(x) \\
& \left(-\frac{d}{d x}+\frac{x}{2}\right) h e_{m}(x)=\sqrt{m+1} h e_{m+1}(x)
\end{aligned}
$$

so, we can set the following shift operators [14-17]:

$$
\widehat{a}_{+}=\left(-\frac{d}{d x}+\frac{x}{2}\right), \widehat{a}_{-}=\left(\frac{d}{d x}+\frac{x}{2}\right)
$$

and then we can rewrite the above relation in a formal way:

$$
\widehat{a}_{+} h e_{n}(x)=\sqrt{n+1} h e_{n+1}(x), \quad \widehat{a}_{-} h e_{n}(x)=\sqrt{n} h e_{n-1}(x)
$$

The above operators are not dependent on discrete parameters so not to change with the indexes of the functions and then the following relation holds:

$$
\widehat{a}_{+} \widehat{a}_{-} h e_{n}(x)=n h e_{n}(x)
$$

We can note that the one-variable orthogonal Hermite functions $h e_{m}(x)$ solved the following ordinary differential equations:

$$
\left[\frac{d^{2}}{d x^{2}}-\frac{x^{2}}{4}+\left(m+\frac{1}{2}\right)\right] h e_{m}(x)=0
$$

In fact, by expliciting the previous operatorial relation, we have:

$$
\left(-\frac{d}{d x}+\frac{x}{2}\right)\left(\frac{d}{d x}+\frac{x}{2}\right) h e_{m}(x)=m h e_{m}(x)
$$

then:

$$
\left(-\frac{d^{2}}{d x^{2}}-\frac{x}{2} \frac{d}{d x}-\frac{1}{2}+\frac{x}{2} \frac{d}{d x}+\frac{x^{2}}{4}\right) h e_{m}(x)=m h e_{m}(x)
$$

and finally:

$$
\left(-\frac{d^{2}}{d x^{2}}+\frac{x^{2}}{4}-\frac{1}{2}-m\right) h e_{m}(x)=0
$$

By the definition of the one-variable Hermite functions $h e_{n}(x)$, we can now generalize the above results that were obtained using the ordinary one-variable Hermite polynomials and applying them to the two-variable Hermite polynomials. We remind that the explicit forms of the generalized Hermite polynomials of type $H_{n}(x, y)$ reads [2]:

$$
H_{n}(x, y)=\sum_{r=0}^{[n / 2]} \frac{n !}{r !(n-2 r) !}(-y)^{r}(2 x)^{n-2 r}
$$

Accordingly, it is also possible to define analogous Hermite functions of two variables that are orthogonal, by using the expression of the generalized two-variable Hermite polynomials $[10,14]$. 
Definition 2. Let $x$ and $y$ be two real variables and $h e_{n}(x)$ be the one-variable Hermite function. We define the two-variable Hermite function $e_{n}(x, y)$, given by the expression:

$$
h e_{n}(x, y)=\sum_{r=0}^{[n / 2]} \sqrt{\frac{n !}{r !(n-2 r) !}} h e_{n-2 r}(x) h e_{r}(y)
$$

Proposition 1. The two-variable Hermite functions $h e_{m}(x, y)$ are orthogonal functions on the interval $(-\infty,+\infty)$.

Proof. We have to prove that the following integral:

$$
\int_{-\infty}^{+\infty} d y \int_{-\infty}^{+\infty} h e_{n}(x, y) h e_{m}(x, y) d x
$$

is a finite number, thus the functions are orthogonal. By substituting the explicit expression of the two-variable Hermite functions $h e_{m}(x, y)$, as given in the previous definition, we get:

$$
\begin{aligned}
& \int_{-\infty}^{+\infty} d y \int_{-\infty}^{+\infty} h e_{n}(x, y) h e_{m}(x, y) d x= \\
& \sum_{r=0}^{[n / 2]} \sum_{s=0}^{[m / 2]} \sqrt{\frac{n ! m !}{(n-2 r) !(m-2 s) ! r ! s !}} \int_{-\infty}^{+\infty} h e_{n-2 r}(x) h e_{m-2 s}(x) d x \int_{-\infty}^{+\infty} h e_{r}(y) h e_{s}(y) d y
\end{aligned}
$$

and since the one-variable Hermite functions are orthonormal on the interval $(-\infty,+\infty)$, we have:

$$
\begin{aligned}
& \int_{-\infty}^{+\infty} d y \int_{-\infty}^{+\infty} h e_{n}(x, y) h e_{m}(x, y) d x= \\
& \quad=\sum_{r=0}^{[n / 2]} \sum_{s=0}^{[m / 2]} \sqrt{\frac{n ! m !}{(n-2 r) !(m-2 s) ! r ! s !}} \int_{-\infty}^{+\infty} h e_{n-2 r}(x) h e_{m-2 s}(x) d x \delta_{r, s} .
\end{aligned}
$$

We note that, in the above summations, all of the terms are zero, except when $r=s$, and then we can rewrite the previous relation in the form:

$$
\begin{aligned}
& \int_{-\infty}^{+\infty} d y \int_{-\infty}^{+\infty} h e_{n}(x, y) h e_{m}(x, y) d x= \\
& \quad=\sum_{r=0}^{[n / 2]} \sum_{r=0}^{[m / 2]} \sqrt{\frac{n ! m !}{((n-2 r) !)^{2}(r !)^{2}}} \int_{-\infty}^{+\infty} h e_{n-2 r}(x) h e_{m-2 r}(x) d x
\end{aligned}
$$

and by applying again the orthonormal property of the one-variable Hermite functions $h e_{m}(x)$, we similarly obtain:

$$
\int_{-\infty}^{+\infty} d y \int_{-\infty}^{+\infty} h e_{n}(x, y) h e_{m}(x, y) d x=\sum_{r=0}^{[n / 2]} \sum_{r=0}^{[m / 2]} \sqrt{\frac{n ! m !}{((n-2 r) !)^{2}(r !)^{2}}} \delta_{n, m}
$$

Additionally, in this case, the only non trivial value it is obtained for $n=m$, and so we can conclude with:

$$
\int_{-\infty}^{+\infty} d y \int_{-\infty}^{+\infty} h e_{n}(x, y) h e_{m}(x, y) d x=\sum_{r=0}^{[n / 2]} \frac{n !}{(n-2 r) ! r !} \delta_{n, m}
$$


which proves the orthogonality of the two-variable Hermite functions $h e_{m}(x, y)$.

It could be useful to observe that the term obtained in the above orthogonal relation can be read as a special case of the two-variable Hermite polynomials of the type $H_{n}^{\prime}$, which is:

$$
H_{n}^{\prime}\left(\frac{1}{2},-1\right)=\sum_{r=0}^{[n / 2]} \frac{n !}{r !(n-2 r) !}
$$

We can derive the generating function for the two-variable orthogonal Hermite functions $h e_{m}(x, y)$ by using the structure and the identities of the Hermite polynomials. For this purpose, we use a different class of two-variable Hermite polynomials called $H e_{m}^{\prime}(x, y)$ [2] having the generating function given by:

$$
\exp \left(x t-\frac{t^{2}}{2}+y t^{2}-\frac{t^{4}}{2}\right)=\sum_{m=0}^{+\infty} \frac{t^{m}}{m !} H e_{m}^{\prime}(x, y)
$$

By manipulating the argument of the exponential, we obtain:

$$
\begin{aligned}
& \exp \left(x t-\frac{t^{2}}{2}+y t^{2}-\frac{t^{4}}{2}\right)= \\
& \quad=\exp \left(x t-\frac{t^{2}}{2}\right) \exp \left(y t^{2}-\frac{t^{4}}{2}\right)= \\
& \quad=\sum_{m=0}^{+\infty} \frac{t^{m}}{m !} H e_{m}(x) \sum_{r=0}^{+\infty} \frac{t^{2 r}}{r !} H e_{r}(y)
\end{aligned}
$$

and by setting $m+2 r=n$, after rearranging the indexes in the above summations, we end up with:

$$
H e_{m}^{\prime}(x, y)=m ! \sum_{r=0}^{[m / 2]} \frac{1}{(m-2 r) ! r !} H e_{m-2 r}(x) H e_{r}(y)
$$

which gives an expression of the two-variable Hermite polynomials $H e_{m}^{\prime}(x, y)$ in terms of the ordinary one-variable Hermite polynomials. We will use the relation that is shown above to state the link between the two-variable orthogonal Hermite functions $h e_{m}(x, y)$ and their generating function.

We start substituting in the definition of the functions $h e_{m}(x, y)$ the expression of the one-variable orthogonal Hermite functions $h e_{m}(x)$ :

$$
h e_{m}(x, y)=\sum_{r=0}^{[m / 2]} \sqrt{\frac{m !}{(m-2 r) ! r !}}\left(\frac{1}{\sqrt{2 \pi}(m-2 r) !}\right)^{\frac{1}{2}}\left(\frac{1}{\sqrt{2 \pi} r !}\right)^{\frac{1}{2}} e^{-\frac{x^{2}}{4}} e^{-\frac{y^{2}}{4}} H e_{m-2 r}(x) H e_{r}(y)
$$

which gives:

$$
h e_{m}(x, y)=e^{-\frac{x^{2}}{4}} e^{-\frac{y^{2}}{4}} \frac{1}{\sqrt{2 \pi}} \sqrt{m !} \sum_{r=0}^{[m / 2]} \frac{1}{(m-2 r) ! r !} H e_{m-2 r}(x) H e_{r}(y)
$$

and by substituting the explicit form of the Hermite polynomials $H e_{m}^{\prime}(x, y)$, we have:

$$
h e_{m}(x, y)=\frac{\sqrt{m !}}{\sqrt{2 \pi}} e^{-\frac{x^{2}}{4}} e^{-\frac{y^{2}}{4}} \frac{H e_{m}^{\prime}(x, y)}{m !} .
$$

By expliciting the two-variable Hermite polynomials $H e_{m}^{\prime}(x, y)$ in terms of the Hermite functions $h e_{m}(x, y)$, the previous equation reads:

$$
H e_{m}^{\prime}(x, y)=\frac{m !}{\sqrt{m !}} \sqrt{2 \pi} e^{\frac{x^{2}+y^{2}}{4}} h e_{m}(x, y)
$$


which, once replaced in the expression, allows for us to write:

$$
\exp \left(x t-\frac{t^{2}}{2}+y t^{2}-\frac{t^{4}}{2}\right)=\sum_{m=0}^{+\infty} \frac{t^{m}}{m !}\left[\frac{m !}{\sqrt{m !}} \sqrt{2 \pi} e^{\frac{x^{2}+y^{2}}{4}} h e_{m}(x, y)\right]
$$

and then, we can finally state the expression of the generating function of the two-variable Hermite functions $h e_{m}(x, y)$ :

$$
\frac{1}{\sqrt{2 \pi}} e^{-\frac{1}{2}(x-t)-\frac{1}{2}\left(y-t^{2}\right)} e^{\frac{x^{2}+y}{4}}=\sum_{m=0}^{+\infty} \frac{t^{m}}{\sqrt{m !}} h e_{m}(x, y) .
$$

Because the two-variable Hermite polynomials satisfy many interesting identities, it is possible to derive similar relations for the two-variable Hermite functions $h e_{n}(x, y)$.

The starting point is the link between the polynomials $H e_{m}^{\prime}(x, y)$ and the functions $h e_{m}(x, y)$ showed above. By deriving with respect to $x$ in the previous relation, we have:

$$
\frac{\partial}{\partial x} h e_{m}(x, y)=\frac{1}{\sqrt{2 \pi} m !} e^{-\frac{y^{2}}{4}}\left[-\frac{x}{2} e^{-\frac{x^{2}}{4}} H e_{m}^{\prime}(x, y)+e^{-\frac{x^{2}}{4}} \frac{\partial}{\partial x} H e_{m}^{\prime}(x, y)\right]
$$

and then:

$$
\frac{\partial}{\partial x} h e_{m}(x, y)=-\frac{1}{\sqrt{2 \pi} m !} \frac{x}{2} e^{-\frac{x^{2}+y^{2}}{4}} H e_{m}^{\prime}(x, y)+\frac{1}{\sqrt{2 \pi} m !} e^{-\frac{x^{2}+y^{2}}{4}} m H e_{m-1}^{\prime}(x, y)(x, y)
$$

to conclude with the following generalization:

$$
\frac{\partial}{\partial x} h e_{m}(x, y)=-\frac{x}{2} h e_{m}(x, y)+\sqrt{m} h e_{m-1}(x, y) .
$$

In the same way, it is possible to state an analogous recurrence relation satisfied by the Hermite functions $h e_{m}(x, y)$. In fact, by deriving now with respect to $y$ in the same equation of above, we obtain:

$$
\frac{\partial}{\partial y} h e_{m}(x, y)=\frac{1}{\sqrt{2 \pi} m !} e^{-\frac{x^{2}}{4}}\left[-\frac{y}{2} e^{-\frac{x^{2}}{4}} H e_{m}^{\prime}(x, y)+e^{-\frac{y^{2}}{4}} \frac{\partial}{\partial y} H e_{m}^{\prime}(x, y)\right]
$$

which gives:

$$
\frac{\partial}{\partial y} h e_{m}(x, y)=-\frac{1}{\sqrt{2 \pi} m !} \frac{y}{2} e^{-\frac{x^{2}+y^{2}}{4}} H e_{m}^{\prime}(x, y)+\frac{1}{\sqrt{2 \pi} m !} e^{-\frac{x^{2}+y^{2}}{4}} m(m-1) H e_{m-2}^{\prime}(x, y) .
$$

By using the expression of the Hermite polynomials of the form $H e_{m}^{\prime}(x, y)$, we can finally state the second generalized recurrence relation for the two-variable Hermite functions $h e_{m}(x, y)$ :

$$
\frac{\partial}{\partial y} h e_{m}(x, y)=-\frac{y}{2} h e_{m}(x, y)+\sqrt{m(m-1)} h e_{m-2}(x, y) .
$$

A further recurrence relation involving the Hermite functions $h e_{m}(x, y)$ can be deduced by operating directly in the equation linking the generalized Hermite polynomials of the type $\mathrm{He}_{m}^{\prime}(x, y)$ and its generating function. We remind, once again, that the generating function of the polynomials $H e_{m}^{\prime}(x, y)$ has the following expression:

$$
\exp \left(x t-\frac{t^{2}}{2}+y t^{2}-\frac{t^{4}}{2}\right)=\sum_{m=0}^{+\infty} \frac{t^{m}}{m !} H e_{m}^{\prime}(x, y)
$$


and by deriving both sides with respect to $t$, we obtain:

$$
\left(x-t+2 y t-2 t^{3}\right) \sum_{m=0}^{+\infty} \frac{t^{m}}{m !} H e_{m}^{\prime}(x, y)=\sum_{m=0}^{+\infty} m \frac{t^{m-1}}{m !} H e_{m}^{\prime}(x, y) .
$$

By exploiting the terms in the above relation, we can write:

$$
\begin{aligned}
& x \sum_{m=0}^{+\infty} \frac{t^{m}}{m !} H e_{m}^{\prime}(x, y)-\sum_{m=0}^{+\infty} \frac{t^{m+1}}{m !} H e_{m}^{\prime}(x, y)+ \\
& +2 y \sum_{m=0}^{+\infty} \frac{t^{m+1}}{m !} H e_{m}^{\prime}(x, y)-2 \sum_{m=0}^{+\infty} \frac{t^{m+3}}{m !} H e_{m}^{\prime}(x, y)= \\
& =\sum_{m=0}^{+\infty} m \frac{t^{m-1}}{m !} H e_{m}^{\prime}(x, y)
\end{aligned}
$$

and by equating the terms of the same power of $m$, we have:

$$
x \frac{H e_{m}^{\prime}(x, y)}{m !}+(2 y-1) \frac{H e_{m-1}^{\prime}(x, y)}{(m-1) !}-2 \frac{H e_{m-3}^{\prime}(x, y)}{(m-3) !}=\frac{m+1}{(m+1) !} H e_{m+1}^{\prime}(x, y)
$$

which gives the important recurrence relation for the generalized Hermite polynomials $H e_{m}^{\prime}(x, y)$ :

$$
x H e_{m}^{\prime}(x, y)+(2 y-1) m H e_{m-1}^{\prime}(x, y)-2[m(m-1)(m-2)] H e_{m-3}^{\prime}(x, y)=H e_{m+1}^{\prime}(x, y)
$$

We can use the relation that is stated above to derive the analogous identity for the two-variable Hermite functions $h e_{m}(x, y)$. In fact, by substituting the expression of the Hermite polynomials $H e_{m}^{\prime}(x, y)$ in terms of the Hermite functions $h e_{m}(x, y)$, we have:

$$
\begin{aligned}
x \sqrt{m !} & \sqrt{2 \pi} e^{\frac{x^{2}+y^{2}}{4}} h e_{m}(x, y)+(2 y-1) m \sqrt{(m-1) !} \sqrt{2 \pi} e^{\frac{x^{2}+y^{2}}{4}} h e_{m-1}(x, y)+ \\
& -2[m(m-1)(m-2)] \sqrt{(m-3) !} \sqrt{2 \pi} e^{\frac{x^{2}+y^{2}}{4}} h e_{m-3}(x, y)= \\
& =\sqrt{(m+1) !} \sqrt{2 \pi} e^{\frac{x^{2}+y^{2}}{4}} h e_{m+1}(x, y)
\end{aligned}
$$

and we can finally conclude with (as discussed in [18]):

$$
\begin{aligned}
& x h e_{m}(x, y)+(2 y-1) \sqrt{m} h e_{m-1}(x, y)-2 \sqrt{m(m-1)(m-2)} h e_{m-3}(x, y)= \\
& =\sqrt{m+1} h e_{m+1}(x, y)
\end{aligned}
$$

\section{Relevant Relations Involving Bi-Orthogonal Hermite Functions}

In the previous sections, we have introduced the one-variable, one-index Hermite polynomials $H_{n}(x)$, and their generalization $H_{n}(x, y)$. It is possible to use the polynomials $H_{n}(x)$ to introduce a different class of Hermite polynomials with two indexes and two variables, which are a vectorial extension (as discussed in [18]); this means that from an index acts on a one-dimensional variable, we will have a couple of indexes acting on a two-dimensional variable:

$$
H_{n}(x) \rightarrow H_{m, n}(x, y)
$$

Let the positive quadratic form:

$$
q(x, y)=a x^{2}+2 b x y+c y^{2}, \quad a, c>0, \quad \Delta=a c-b^{2}>0
$$


where $a, b, c$ are real numbers and the associated matrix reads:

$$
\widehat{M}=\left(\begin{array}{ll}
a & b \\
b & c
\end{array}\right), \quad|\widehat{M}|>0
$$

and by considering a vector $\underline{z}=\left(\begin{array}{l}x \\ y\end{array}\right)$ in the space $\mathbb{R}^{2}$, it follows:

$$
\begin{gathered}
q(\underline{z})=\underline{z}^{t} \widehat{M} \underline{z} \\
q(\underline{z})=\left(\begin{array}{ll}
x & y
\end{array}\right)\left(\begin{array}{ll}
a & b \\
b & c
\end{array}\right)\left(\begin{array}{l}
x \\
y
\end{array}\right)=a x^{2}+2 b x y+c y^{2}
\end{gathered}
$$

Definition 3. We will call two-index, two-variable Hermite polynomials, indicated with the symbol $H_{m, n}(x, y)$, the polynomials defined by the following generating function:

$$
e^{\underline{z}^{t} \widehat{M} \underline{h}-\frac{1}{2} \underline{h}^{t} \widehat{M} \underline{h}}=\sum_{m=0}^{+\infty} \sum_{n=0}^{+\infty} \frac{t^{m}}{m !} \frac{u^{n}}{n !} H_{m, n}(x, y)
$$

where:

$$
\underline{z}=\left(\begin{array}{l}
x \\
y
\end{array}\right), \underline{h}=\left(\begin{array}{l}
t \\
u
\end{array}\right) \in \mathbb{R}^{2} \text { with } t \neq u,(|t|,|u|)<+\infty
$$

These polynomials are exploited in many fields of pure and applied mathematics, they are very useful in the description of the quantum treatment of coupled harmonic oscillator [19].

By using the definition of the quadratic form, we can introduce the related adjoint class of these polynomials, by setting:

$$
\bar{q}(\underline{z})=\underline{z}^{t} \widehat{M}^{-1} \underline{z}
$$

we have

$$
e^{\underline{\underline{v}} \underline{\underline{M}}^{t} \widehat{M}^{-1} \underline{k}-\frac{1}{2} \underline{k}^{t} \widehat{M}^{-1} \underline{k}}=\sum_{m=0}^{+\infty} \sum_{n=0}^{+\infty} \frac{r^{m}}{m !} \frac{s^{n}}{n !} G_{m, n}(x, y)
$$

where:

$$
\underline{v}=\left(\begin{array}{l}
\xi \\
\eta
\end{array}\right), \underline{k}=\left(\begin{array}{l}
r \\
s
\end{array}\right) \in \mathbb{R}^{2} \text { with } \underline{v}=\widehat{M} \underline{z} \text { and } r \neq s,(|r|,|s|)<+\infty
$$

The expression of the generating function defining the adjoint Hermite polynomials of two-index and two-variable $G_{m, n}(x, y)$, could be recast in the following form:

$$
e^{z^{t} \underline{k}-\frac{1}{2} \underline{k}^{t} \widehat{M}^{-1} \underline{k}}=\sum_{m=0}^{+\infty} \sum_{n=0}^{+\infty} \frac{r^{m}}{m !} \frac{s^{n}}{n !} G_{m, n}(x, y)
$$

The above introduced vectorial Hermite polynomials satisfy, with their adjoints, many and relevant properties. It is now interesting to explore the possibility to find similar Hermite functions as those that are defined in the previous section, in order to obtain an extension of the concepts and the related identities satisfied from the Hermite polynomials $H_{m, n}(x, y)$ and their adjoints $G_{m, n}(x, y)$.

The structure of the vectorial extension Hermite polynomials is based on the fact that a vector index acts on a vector variable or, which is the same, a couple of indexes act on a couple of variables. This suggests that we can not expect the same relation linking the two-index, two-variable Hermite polynomials $H_{m, n}(x, y)$ and $G_{m, n}(x, y)$, and the related Hermite functions we are going to define; moreover, the concept of orthogonality is not the same as the existing one for the one-index Hermite polynomials of type $H_{n}(x)$ and $H_{n}(x, y)$. The two-index, two-variable Hermite polynomials $H_{m, n}(x, y)$ 
and their adjoints $G_{m, n}(x, y)$ satisfy the following bi-orthogonality condition (result already discussed in detail in [18]):

$$
\int_{-\infty}^{+\infty} d y \int_{-\infty}^{+\infty} H_{m, n}(x, y) G_{r, s}(x, y) e^{-\frac{1}{2} z^{t} \widehat{M} z} d x=\frac{2 \pi}{\sqrt{\Delta}} m ! n ! \delta_{m, r} \delta_{n, s}
$$

Remark 3. The proof is based on the definition of the generating functions and use the vectorial techniques of differentiation. The weight function:

$$
e^{-\frac{1}{2} \underline{z}^{t} \widehat{M} \underline{z}}
$$

is easily recognized similar to the related weight function for the ordinary Hermite polynomials $H_{n}(x)$. We can use the above result to define functions that are based on the two-index, two-variable Hermite polynomials.

Definition 4. Let the Hermite polynomials $H_{m, n}(x, y)$ and $G_{m, n}(x, y)$ we call two-index, two-variable Hermite functions, be the functions defined in the following way:

$$
\begin{aligned}
\bar{H}_{m, n}(x, y) & =\frac{\sqrt[4]{\Delta}}{2 \pi} \frac{1}{\sqrt{m ! n !}} H_{m, n}(x, y) e^{-\frac{1}{4} \underline{z}^{t} \widehat{M}^{-1} \underline{z}} \\
\bar{G}_{m, n}(x, y) & =\frac{\sqrt[4]{\Delta}}{2 \pi} \frac{1}{\sqrt{m ! n !}} G_{m, n}(x, y) e^{-\frac{1}{4} \underline{z}^{t} \widehat{M}^{-1} \underline{z}}
\end{aligned}
$$

which are obviously bi-orthonormal.

We see how to extend the differential relations showed for the polynomials $H_{n}(x)$ ti emphasize the relevance of the bi-orthogonal Hermite functions.

We start to observe that the Hermite polynomials of type $H_{m, n}(x, y)$ and $G_{m, n}(x, y)$ solve the following partial differential equation:

$$
\frac{\partial}{\partial \tau} S_{m, n}(x, y ; \tau)=-\frac{1}{2}\left(\begin{array}{ll}
\partial_{x} & \partial_{y}
\end{array}\right) \widehat{M}^{-1}\left(\begin{array}{l}
\partial_{x} \\
\partial_{y}
\end{array}\right) S_{m, n}(x, y ; \tau)
$$

satisfying the conditions at $\tau=0$

$$
S_{m, n}(x, y ; 0)=\left\{\begin{array}{l}
\xi^{m} \eta^{n}, \text { when } S_{m, n}=H_{m, n} \\
x^{m} y^{n}, \text { when } S_{m, n}=G_{m, n}
\end{array}\right.
$$

Remark 4. The proof is an immediate consequence of the properties satisfied by the polynomials $H_{m, n}(x, y)$ and $G_{m, n}(x, y)$, deduced by their structure, while using the similar well-known relations of the ordinary Hermite polynomials, by defining the following shift operators:

$$
\begin{aligned}
& \widehat{a}_{+, 0}=\frac{1}{2}(a x+b y)-\frac{\partial}{\partial x}, \quad \widehat{a}_{-, 0}=\frac{1}{\Delta}\left(c \frac{\partial}{\partial x}-b \frac{\partial}{\partial y}\right)+\frac{1}{2} x \\
& \widehat{a}_{0,+}=\frac{1}{2}(b x+c y)-\frac{\partial}{\partial y}, \quad \widehat{a}_{0,-}=\frac{1}{\Delta}\left(b \frac{\partial}{\partial x}-a \frac{\partial}{\partial y}\right)+\frac{1}{2} y
\end{aligned}
$$

where $\Delta=a c-b^{2}$ is the determinant of the quadratic form that is defined above.

The above operators are free from any parameters, not presenting any index variable in their structure, therefore different from the shift operators related to Hermite polynomials. The action of these operators could be summarized, as follows:

$$
\widehat{a}_{+, 0} \bar{H}_{m, n}(x, y)=\sqrt{m+1} \bar{H}_{m+1, n}, \quad \widehat{a}_{-, 0} \bar{H}_{m, n}(x, y)=\sqrt{m} \bar{H}_{m-1, n}(x, y)
$$




$$
\widehat{a}_{0,+} \bar{H}_{m, n}(x, y)=\sqrt{n+1} \bar{H}_{m, n+1}, \quad \widehat{a}_{0,-} \bar{H}_{m, n}(x, y)=\sqrt{n} \bar{H}_{m, n-1}(x, y)
$$

By virtue of the above relations, we can state the important result concerning the partial differential equation that is solved by the bi-orthogonal Hermite functions [18]:

$$
\left[-\underline{\partial}_{z}^{t} \widehat{M}^{-1} \underline{\partial}_{z}-\left(m+n+1-\frac{1}{4} \underline{z}^{t} \widehat{M} \underline{z}\right)\right] \bar{H}_{m, n}(x, y)=0
$$

where $\underline{z}=\left(\begin{array}{l}x \\ y\end{array}\right)$ and $\underline{\partial}_{z}=\left(\begin{array}{c}\frac{\partial}{\partial x} \\ \frac{\partial}{\partial y}\end{array}\right)$

Remark 5. To prove the statement, we use the following operational relations:

$$
\begin{aligned}
& \widehat{a}_{+, 0}\left[\widehat{a}_{-, 0} \bar{H}_{m, n}(x, y)\right]=m \bar{H}_{m, n}(x, y) \\
& \widehat{a}_{0,+}\left[\widehat{a}_{0,-} \bar{H}_{m, n}(x, y)\right]=n \bar{H}_{m, n}(x, y)
\end{aligned}
$$

which emphasizes the similarity with the orthogonality relation previous established for the ordinary Hermite polynomials:

$$
\widehat{a}_{+} \widehat{a}_{-} h e_{n}(x)=n h e_{n}(x)
$$

To state the analogous result for the functions $\bar{G}_{m, n}(x, y)$ it is sufficient to remind that the adjoint quadratic form:

$$
\bar{q}(\underline{z})=\underline{z}^{t} \widehat{M}^{-1} \underline{z}
$$

which introduced the variable:

$$
\underline{v}=\widehat{M} \underline{z} \text {, where } \underline{v}=\left(\begin{array}{l}
\xi \\
\eta
\end{array}\right)
$$

\section{Further Investigations}

The previous discussed Hermite polynomials and related bi-orthogonal functions satisfy a plethora of relevant relations, for instance in [10,14,20-22], but it is much more interesting to explore how the particular bi-orthogonality relation could be extend to other families of classical orthogonal polynomials [23,24]. Note that ordinary and generalized Laguerre and Legendre polynomials [25-27], for example, as well as Chebyshev polynomials [28-32], could be expressed in terms of polynomials recognized as Hermite. Regarding the generalized Laguerre polynomials:

$$
{ }_{2} L_{n}(x, y)=H_{n}\left(y, \widehat{D}_{x}^{-1}\right)
$$

where: ${ }_{2} L_{n}(x, y)=n ! \sum_{r=0}^{[n / 2]} \frac{y^{n-2 r} x^{r}}{(n-2 r) !(r !)^{2}}, \widehat{D}_{x}^{-1}$ the inverse of derivative operator for the Legendre polynomials, it holds:

$$
{ }_{2} L_{n}(x, y)\left(-\frac{1}{4}\left(1-y^{2}\right), y\right)=P_{n}(y)
$$

where:

$$
P_{n}(y)=n ! \sum_{r=0}^{[n / 2]} \frac{(-1)^{n-2 r} y^{r}\left(1-y^{2}\right)^{n-2 r}}{(n-2 r) !(r !)^{2} 2^{2(n-2 r)}}
$$

Regarding the Chebyshev polynomials of first and second kind, for $t \in \mathbb{R}$, we have:

$$
U_{n}(x)=\frac{1}{n !} \int_{0}^{+\infty} e^{-t} t^{n} H_{n}\left(2 x,-\frac{1}{t}\right) d t
$$




$$
T_{n}(x)=\frac{1}{2(n-1) !} \int_{0}^{+\infty} e^{-t} t^{n-1} H_{n}\left(2 x,-\frac{1}{t}\right) d t
$$

or, more in general:

$$
U_{n}(x, y)=\frac{1}{n !} \int_{0}^{+\infty} e^{-t} t^{n} H_{n}\left(x, \frac{y}{t}\right) d t
$$

and, furthermore:

$$
U_{n}(x, y)^{(m)}=\frac{1}{n !} \int_{0}^{+\infty} e^{-t} t^{n} H_{n}^{(m)}\left(x, \frac{y}{t}\right) d t
$$

where

$$
U_{n}(x, y)^{(m)}=\sum_{r=0}^{[n / m]} \frac{(n-r) ! x^{n-m r} y^{r}}{r !(n-m r) !}, \quad H_{n}^{(m)}(x, y)=\sum_{r=0}^{[n / m]} \frac{n ! x^{n-m r} y^{r}}{r !(n-m r) !}
$$

The above relations are some examples of the plethora of generalizations for many families of orthogonal polynomials and special functions; this emphasizes the powerful tool that is represented by the wide family of those polynomials recognized as Hermite polynomials.

Before concluding, it is worth noting that the bivariate orthogonal Hermite polynomials [33]:

$$
H_{n, m}(x, y ; \Lambda)=\sum_{k=0}^{\min (n, m)}(-1)^{k} k !\left(\begin{array}{c}
m \\
k
\end{array}\right)\left(\begin{array}{l}
n \\
k
\end{array}\right) a^{(n-k) / 2} b^{k} c^{(m-k) / 2} H_{n-k}^{e}\left(\frac{a x+b y}{\sqrt{a}}\right) H_{m-k}^{e}\left(\frac{b x+c y}{\sqrt{c}}\right)
$$

present the same structure of the two-index, two-variable Hermite polynomials defined in Section 4 as well as other parabolic polynomials, recognized as belonging to the Hermite family, showed by A. Wünsche [34]. In a forthcoming article, we can deep discuss the analogy and differences between these different classes of Hermite polynomials.

Funding: This research received no external funding.

Conflicts of Interest: The authors declare no conflict of interest.

\section{References}

1. Appell, P.; De Fériet, J.K. Fonctions hypergéométriques et hypersphériques: Polynomes d'Hermite; Gauthier-Villars: Paris, France, 1926.

2. Gould, H.W.; Hopper, A. Operational formulas connected with two generalizations of Hermite polynomials. Duke Math. J. 1962, 29, 51-63. [CrossRef]

3. Koornwinder, T.H. Orthogonal Polynomials in Two Variables Which Are Eigenfunctions of Two Independent Partial Differential Operators, 2: Prepublication; Stichting Mathematisch Centrum. Toegepaste Wiskunde TW 137/73; Stichting Mathematisch Centrum: Amsterdam, The Netherlands, 1973; Volume 77.

4. Koornwinder, T.H. Orthogonal Polynomials in Two Variables Which Are Eigenfunctions of Two Algebraically Independent Partial Differential Operators. III; Indagationes Mathematicae (Proceedings); Elsevier: Amsterdam, The Netherlands, 1974; Volume 36, pp. 357-369.

5. Cesarano, C.; Cennamo, G.M.; Placidi, L. Operational methods for Hermite polynomials with applications. rn 2014, 2, 2 .

6. Bertola, M.; Gekhtman, M.; Szmigielski, J. Cauchy biorthogonal polynomials. J. Approx. Theory 2010, 162, 832-867. [CrossRef]

7. Bertola, M. Biorthogonal polynomials for two-matrix models with semiclassical potentials. J. Approx. Theory 2007, 144, 162-212. [CrossRef]

8. Bertola, M.; Eynard, B.; Harnad, J. Duality, Biorthogonal Polynomials and Multi-Matrix Models. Commun. Math. Phys. 2002, 229, 73-120. [CrossRef]

9. Bertola, M.; Gekhtman, M.; Szmigielski, J. Cubic string boundary value problems and Cauchy biorthogonal polynomials. J. A Phys. Math. Theor. 2009, 42, 454006 [CrossRef]

10. Cesarano, C. Operational methods and new identities for Hermite polynomials. Math. Model. Nat. Phenom. 2017, 12, 44-50. [CrossRef] 
11. Srivastava, H.; Manocha, H. Treatise on Generating Functions; John Wiley \& Sons, Inc.: New York, NY, USA, 1984.

12. Dattoli, G.; Ottaviani, P.; Torre, A.; Vázquez, L. Evolution operator equations: Integration with algebraic and finitedifference methods. Applications to physical problems in classical and quantum mechanics and quantum field theory. La Riv. Del Nuovo Cimento (1978-1999) 1997, 20, 3. [CrossRef]

13. Dattoli, G. Hermite-Bessel and Laguerre-Bessel functions: A by-product of the monomiality principle. In Proceedings of the Melfi School on Advanced Topics in Mathematics and Physics, Melfi, Italy, 9-12 May 1999.

14. Dattoli, G.; Cesarano, C. On a new family of Hermite polynomials associated to parabolic cylinder functions. Appl. Math. Comput. 2003, 141, 143-149. [CrossRef]

15. Dattoli, G.; Ricci, P.; Cesarano, C. A note on multi-index polynomials of Dickson type and their applications in quantum optics. J. Comput. Appl. Math. 2002, 145, 417-424. [CrossRef]

16. Dattoli, G.; Lorenzutta, S.; Cesarano, C.; Ricci, P. Second level exponentials and families of Appell polynomials. Integral Transform. Spec. Funct. 2002, 13, 521-527. [CrossRef]

17. Dattoli, G.; Lorenzutta, S.; Ricci, P.; Cesarano, C. On a family of hybrid polynomials. Integral Transform. Spec. Funct. 2004, 15, 485-490. [CrossRef]

18. Cesarano, C.; Fornaro, C.; Vazquez, L. Operational results in bi-orthogonal Hermite functions. Acta Math. Univ. Comen. 2016, 85, 43-68.

19. Kim, Y.S.; Noz, M.E. Phase Space Picture of Quantum Mechanics: Group Theoretical Approach; World Scientific: Singapore, 1991; Volume 40.

20. Dattoli, G.; Lorenzutta, S.; Cesarano, C. Bernstein Polynomials and Operational Methods. J. Comput. Anal. Appl. 2016, 8, 369-377.

21. Cesarano, C. Multi-dimensional Chebyshev polynomials: A non-conventional approach. Commun. Appl. Ind. Math. 2019, 10, 1-19. [CrossRef]

22. Dattoli, G.; Ricci, P.E.; Cesarano, C. The Bessel functions and the Hermite polynomials from a unified point of view. Appl. Anal. 2001, 80, 379-384. [CrossRef]

23. Cesarano, C.; Cennamo, G.M.; Placidi, L. Humbert polynomials and functions in terms of Hermite polynomials towards applications to wave propagation. WSEAS Trans. Math. 2014, 13, 595-602.

24. Cesarano, C.; Assante, D. A note on generalized Bessel functions. Int. J. Math. Model. Methods Appl. Sci. 2013, 7, 625-629.

25. Dattoli, G.; Srivastava, H.M.; Cesarano, C. The Laguerre and Legendre polynomials from an operational point of view. Appl. Math. Comput. 2001, 124, 117-127. [CrossRef]

26. Cesarano, C.; Ricci, P.E. The Legendre polynomials as a basis for Bessel functions. Int. J. Pure Appl. Math. 2016, 111, 129-139. [CrossRef]

27. Cesarano, C.; Germano, B.; Ricci, P. Laguerre-type Bessel functions. Integral Transform. Spec. Funct. 2005, 16, 315-322. [CrossRef]

28. Cesarano, C. Identities and generating functions on Chebyshev polynomials. Georgian Math. J. 2012, 19, 427-440. [CrossRef]

29. Cesarano, C. Generalized Chebyshev polynomials. Hacet. J. Math. Stat. 2014, 43, 731-740.

30. Cesarano, C. Integral representations and new generating functions of Chebyshev polynomials. Hacet. J. Math. Stat 2015, 44, 535-546. [CrossRef]

31. Cesarano, C.; Pinelas, S.; Ricci, P.E. The third and fourth kind pseudo-Chebyshev polynomials of half-integer degree. Symmetry 2019, 11, 274. [CrossRef]

32. Cesarano, C.; Fornaro, C. A note on two-variable Chebyshev polynomials. Georgian Math. J. 2017, 24, 339-349. [CrossRef]

33. Dimitrov, D.; Godoy, E. Zero sets of bivariate Hermite polynomials. J. Math. Anal. Appl. 2015, 421, 830-841.

34. Wünsche, A. Generalized Hermite polynomials associated with functions of parabolic cylinder. Appl. Math. Comput. 2003, 141, 197-213. [CrossRef]

(C) 2020 by the author. Licensee MDPI, Basel, Switzerland. This article is an open access article distributed under the terms and conditions of the Creative Commons Attribution (CC BY) license (http:/ / creativecommons.org/licenses/by/4.0/). 